\title{
Early Blood Pressure Lowering Does Not Reduce Growth of Intraventricular Hemorrhage following Acute Intracerebral Hemorrhage: Results of the INTERACT Studies
}

\author{
Edward Chan ${ }^{a, b} \quad$ Craig S. Anderson ${ }^{a, b} \quad$ Xia Wang ${ }^{a, b}$ Hisatomi Arima ${ }^{a, b}$ \\ Anubhav Saxena ${ }^{a, b}$ Tom J. Moullaali ${ }^{a}$ Candice Delcourt ${ }^{a}$ b Guojun Wu ${ }^{c}$ \\ Jinchao Wang ${ }^{c}$ Guofang Chen $^{d}$ Pablo M. Lavados ${ }^{e}$ f Christian Stapf $^{g}$ \\ Thompson Robinson $^{\mathrm{h}}$ John Chalmers ${ }^{\mathrm{a}}$ b The INTERACT Investigators \\ ${ }^{a}$ The George Institute for Global Health, Royal Prince Alfred Hospital, and ${ }^{b}$ Central Clinical \\ School, University of Sydney, Sydney, N.S.W., Australia; ' Department of Neurology, Hebei \\ Yutian Hospital, Tangshan, and ${ }^{\mathrm{d} D e p a r t m e n t}$ of Neurology, Xuzhou Central Hospital, \\ Xuzhou, China; e Servicio de Neurología, Departamento de Medicina, Clínica Alemana, \\ Universidad del Desarrollo, and fDepartmento de Ciencias Neurológicas, Facultad de \\ Medicina, Universidad de Chile, Santiago, Chile; ${ }^{9} \mathrm{CRCHUM}$, Université de Montréal et \\ Neurologie Vasculaire, Hôpital Notre-Dame - CHUM, Montréal, Que., Canada; \\ ${ }^{\mathrm{h}}$ Department of Cardiovascular Sciences and NIHR Biomedical Research Unit in \\ Cardiovascular Disease, University of Leicester, Leicester, UK
}

Key Words

Stroke · Intracerebral hemorrhage $\cdot$ Intraventricular hemorrhage $\cdot$ Hypertension · Intensive blood pressure lowering

\begin{abstract}
Background: Intraventricular hemorrhage (IVH) extension is common following acute intracerebral hemorrhage (ICH) and is associated with poor prognosis. Aim: To determine whether intensive blood pressure (BP)-lowering therapy reduces IVH growth. Methods: Pooled analyses of the Intensive Blood Pressure Reduction in Acute Cerebral Hemorrhage Trials (INTERACT1 and INTERACT2) computed tomography (CT) substudies; multicenter, open, controlled, randomized trials of patients with acute spontaneous ICH and elevated systolic BP, randomly assigned to intensive $(<140 \mathrm{~mm} \mathrm{Hg})$ or guideline-based $(<180 \mathrm{~mm} \mathrm{Hg})$ BP management. Participants had blinded central analyses of baseline and 24-hour CT. Association of BP lowering to IVH growth was assessed in analysis of covariance. Results: There was no signif-
\end{abstract}


icant difference in adjusted mean IVH growth following intensive $(n=228)$ compared to guideline-recommended $(n=228)$ BP treatment (1.6 versus $2.2 \mathrm{ml}$, respectively; $p=0.56$ ). Adjusted mean IVH growth was nonsignificantly greater in patients with a mean achieved systolic BP $\geq 160 \mathrm{~mm} \mathrm{Hg}$ over $24 \mathrm{~h}(3.94 \mathrm{ml}$; $\mathrm{p}$ trend $=0.26)$. Conclusions: Early intensive BPlowering treatment had no clear effect on IVH in acute ICH.

\section{Introduction}

Approximately one third of patients with acute spontaneous intracerebral hemorrhage (ICH) have intraventricular hemorrhage (IVH) extension, which confers a worse prognosis [1]. High blood pressure (BP) is associated with both hematoma growth and poor outcome [2], but limited data exist on the relationship of BP and IVH [3]. We aimed to determine whether BP-lowering therapy is associated with IVH growth among participants of the pilot and main phases of the Intensive Blood Pressure Reduction in Acute Cerebral Hemorrhage Trials (INTERACT1 and INTERACT2) [2, 4].

\section{Methods}

The INTERACT studies were international, multicenter, prospective, open, blindedendpoint, randomized controlled trials, described in detail elsewhere [2,4]. They involved 3,243 ICH patients ( $<6 \mathrm{~h}$ of onset; 404 INTERACT1, 2,839 INTERACT2) and elevated systolic BP (150-220 mm Hg) randomly assigned to intensive (target systolic BP $<140 \mathrm{~mm} \mathrm{Hg}$ ) or guideline-recommended ( $<180 \mathrm{~mm} \mathrm{Hg}$ ) BP management. In predefined computed tomography (CT) substudies, 1,310 consecutive patients (346 INTERACT1, 964 INTERACT2) had a second CT at $24 \pm 3 \mathrm{~h}$. ICH and IVH volume analyses were performed centrally by expert assessors blind to other data using MIStar version 3.2 (Apollo Medical Imaging Technology). The ethics committees approved the study protocols and written informed consent was obtained from the patients or appropriate surrogates.

Absolute increase in IVH volume was log-transformed to remove skewness and to achieve near-normal distribution of data for analyses. The association of randomized BP-lowering treatment on the absolute increase in IVH volume over $24 \mathrm{~h}$ was assessed in analysis of covariance (ANCOVA), adjusted for age, sex, baseline IVH volume, ICH location and volume, and China region. The mean achieved systolic BP over $24 \mathrm{~h}$ was calculated from the average of 1,6 , 12,18 , and $24 \mathrm{~h}$ postrandomization readings; their association with absolute increase in IVH volume was assessed by ANCOVA adjusting for the same variables, and also for use of statin therapy, baseline systolic BP, and randomized treatment. A p value $<0.05$ was considered statistically significant and SAS version 9.3 (SAS Institute, Cary, N.C., USA) was used in all analyses.

\section{Results}

There were 456 CT substudy participants with IVH and available data (online suppl. fig. 1; for all online suppl. material, see www.karger.com/doi/10.1159/000448897). Guideline-treated patients were more likely to be female, but otherwise there were no significant differences in baseline characteristics, including time from onset to randomization, BP, as well as ICH and IVH volumes (table 1). 

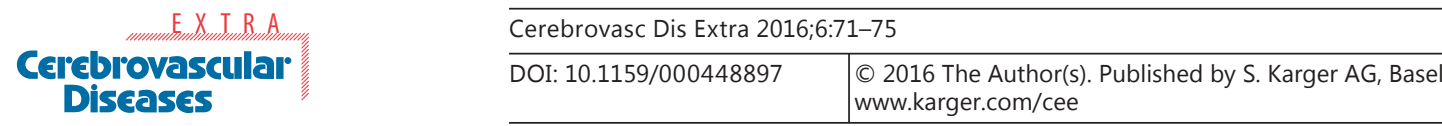

Chan et al.: Early Blood Pressure Lowering Does Not Reduce Growth of Intraventricular Hemorrhage following Acute Intracerebral Hemorrhage

Table 1. Characteristics of patients by randomized treatment

\begin{tabular}{|c|c|c|c|}
\hline Characteristic & $\begin{array}{l}\text { Intensive group } \\
(n=228)\end{array}$ & $\begin{array}{l}\text { Guideline group } \\
(n=228)\end{array}$ & $\begin{array}{l}\mathrm{p} \\
\text { value }\end{array}$ \\
\hline \multicolumn{4}{|l|}{ Demographics } \\
\hline Age, years & $67(13)$ & $68(12)$ & 0.28 \\
\hline Female & $75(33 \%)$ & 97 (43\%) & 0.03 \\
\hline Chinese region & $113(50 \%)$ & $112(49 \%)$ & 0.93 \\
\hline \multicolumn{4}{|l|}{ Medical history } \\
\hline Previous IVH & $15(7 \%)$ & $17(8 \%)$ & 0.72 \\
\hline Previous ischemic stroke & $23(10 \%)$ & $28(12 \%)$ & 0.47 \\
\hline Heart disease & $10(4 \%)$ & $9(4 \%)$ & 0.81 \\
\hline Hypertension & $161(71 \%)$ & $168(74 \%)$ & 0.51 \\
\hline Diabetes mellitus & $32(14 \%)$ & $27(12 \%)$ & 0.47 \\
\hline \multicolumn{4}{|l|}{ Medication history } \\
\hline Antihypertensive agent & $119(52 \%)$ & $118(52 \%)$ & 0.89 \\
\hline Oral anticoagulant & $18(8 \%)$ & $14(6 \%)$ & 0.46 \\
\hline Antiplatelet therapy & $38(17 \%)$ & $40(18 \%)$ & 0.80 \\
\hline Lipid-lowering therapy & $27(12 \%)$ & $26(11 \%)$ & 0.87 \\
\hline \multicolumn{4}{|l|}{ Clinical features } \\
\hline Time from onset to randomization & $3.7(2.7-4.7)$ & $3.5(2.8-4.7)$ & 0.89 \\
\hline Systolic BP & $181(17)$ & $181(17)$ & 0.81 \\
\hline Diastolic BP & $99(15)$ & $100(15)$ & 0.37 \\
\hline Glasgow Coma Scale score ${ }^{1}$ & $14(12-15)$ & $14(12-15)$ & 0.35 \\
\hline NIHSS score ${ }^{2}$ & $14(9-18)$ & $14(9-19)$ & 0.51 \\
\hline NIHSS score $\geq 15$ & $102(45 \%)$ & $113(50 \%)$ & 0.28 \\
\hline \multicolumn{4}{|l|}{ CT findings } \\
\hline Time from onset to first $\mathrm{CT}, \mathrm{h}$ & $1.8(1.3-2.6)$ & $1.8(1.2-2.5)$ & 0.61 \\
\hline Baseline hematoma volume, $\mathrm{ml}$ & $13.3(7.5-27.5)$ & $12.3(7.3-25.7)$ & 0.59 \\
\hline 24-hour hematoma volume, ml & $13.9(7.8-34.0)$ & $13.7(8.0-40.2)$ & 0.36 \\
\hline Baseline IVH volume & $2.5(0.2-9.6)$ & $3.2(0.0-12.6)$ & 0.32 \\
\hline 24-hour IVH volume & $4.3(1.5-12.5)$ & $6.4(1.6-15.3)$ & 0.15 \\
\hline Baseline deep location of hematoma & $186(82 \%)$ & $199(87 \%)$ & 0.09 \\
\hline Baseline intraventricular hematoma extension & $178(78 \%)$ & $171(75 \%)$ & 0.44 \\
\hline
\end{tabular}

No significant differences were observed in absolute or adjusted mean IVH growth between intensive and guideline BP-lowering treatment [3.4 (95\% confidence interval $1.2-5.5)$ vs. $4.8 \mathrm{ml}(2.7-6.9), \mathrm{p}=0.35 ; 1.6(0.2-3.1)$ vs. 2.2 (0.8-3.7), $\mathrm{p}=0.56$, respectively]. Patients with an achieved systolic BP over $24 \mathrm{~h} \geq 160 \mathrm{~mm}$ Hg were younger, more likely to be from China, and had a higher baseline BP (online suppl. table 1). There was a nonsignificant trend towards a greater absolute and adjusted mean IVH growth of $3.58 \mathrm{ml}(1.56-5.72)$ and $3.94 \mathrm{ml}$ (1.46-6.61), respectively, with a mean achieved systolic BP over $24 \mathrm{~h} \geq 160 \mathrm{~mm} \mathrm{Hg}$ (fig. 1). Patients with IVH growth were older, had higher National Institutes of Health Stroke Scale (NIHSS) scores, larger 24-hour ICH volumes, and smaller baseline IVH volumes (online suppl. table 2). The odds ratio for death or major disability was significantly greater with than without IVH growth (adjusted odds ratio 3.51, 95\% confidence interval 2.05-6.02; online suppl. table 3). 

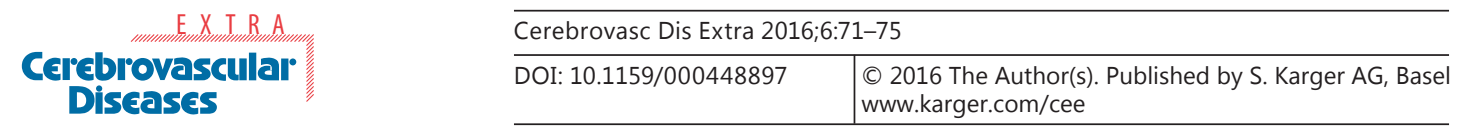

Chan et al.: Early Blood Pressure Lowering Does Not Reduce Growth of Intraventricular Hemorrhage following Acute Intracerebral Hemorrhage

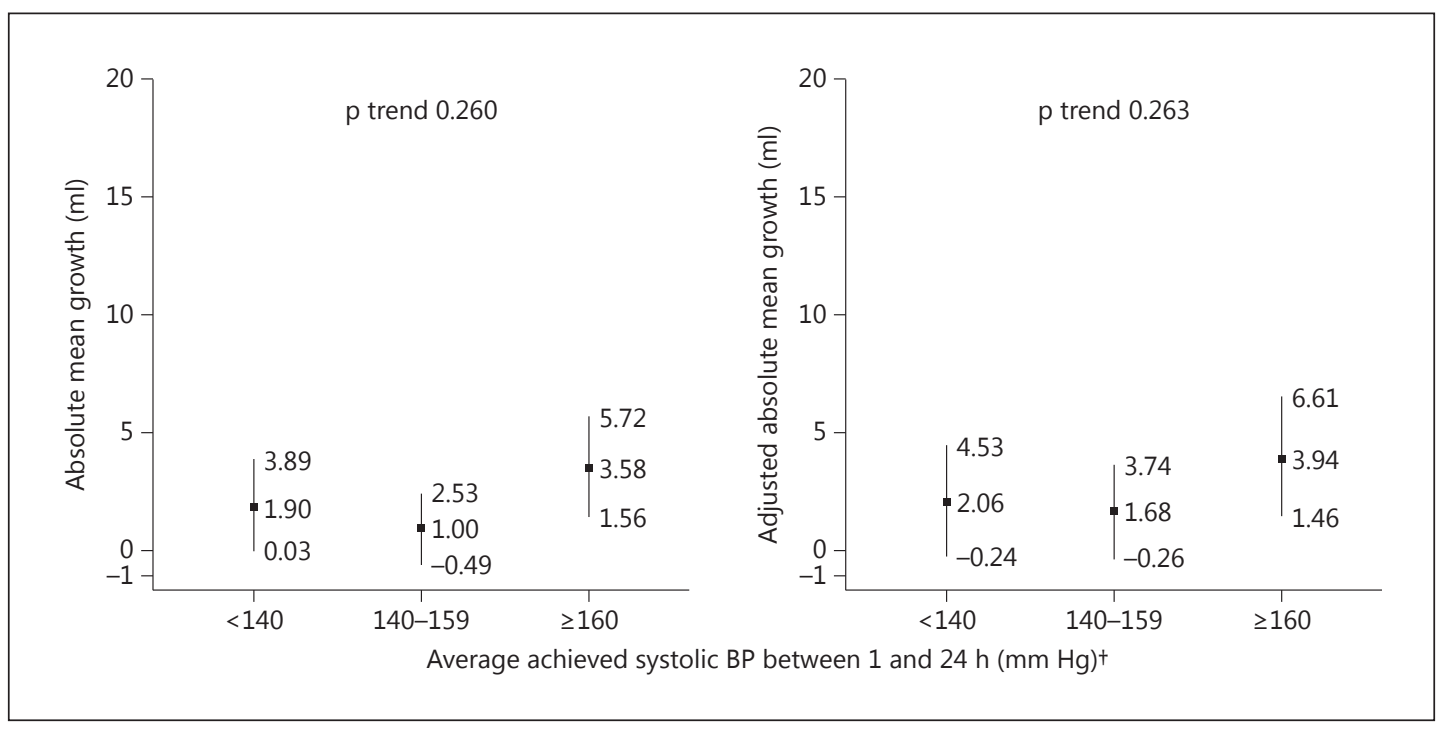

Fig. 1. Intraventricular volume growth by average achieved BP between 1 and $24 \mathrm{~h}$ after randomization. ${ }^{\dagger}$ Assessed by ANCOVA adjusting for age, sex, China region, use of statin, baseline systolic BP, randomized treatment, baseline IVH volume, and ICH location. Because of skewed raw data, adjusted means are reported with $95 \%$ confidence intervals obtained by back-transformation.

\section{Discussion}

This post hoc analysis of 456 IVH patients from the INTERACT CT substudies showed no clear relationship between IVH growth in acute ICH and a strategy of early intensive BP management, supporting an earlier analysis of the INTERACT2 study of no association of BP and IVH volume [1]. Thus, the clinical benefits of intensive BP lowering on functional outcome seen in the INTERACT studies are likely attributable to mechanisms other than IVH expansion.

These results concur with a previous small prospective cohort study, where again no significant difference in admission systolic BP or mean BP across categories of IVH volumes within the first $24 \mathrm{~h}$ of ICH onset was reported [5]. However, the mean 24-hour IVH volume was still higher in our standard BP treatment group, though not significantly different from intensive BP lowering. BP should not be excluded as a contributing factor to IVH growth. Whilst baseline BP per se does not appear to be a major factor in the pathogenesis of IVH, there was a trend towards a greater IVH volume growth at higher average achieved systolic $\mathrm{BP}$ over the first $24 \mathrm{~h}$. Indeed, further analyses of the INTERACT2 study showed that rapid and sustained systolic BP reduction was associated with less ICH hematoma growth over $24 \mathrm{~h} \mathrm{[6].} \mathrm{Another} \mathrm{consideration} \mathrm{relates} \mathrm{to} \mathrm{whether} \mathrm{intensive} \mathrm{BP} \mathrm{reduction} \mathrm{is} \mathrm{associated} \mathrm{with}$ a lower risk of developing IVH. We previously reported that delayed IVH is associated with a worse prognosis, though neither intensive BP lowering nor deep location of ICH were significantly associated with delayed IVH [7].

Whilst the strengths to this study include having a robust, well-characterized cohort from large international trials with standardized care, there are several limitations that include the exclusion of patients with large ICH volumes and impaired Glasgow Coma Scale, which makes the results less generalizable to more severe ICH. Furthermore, despite the large sample size, it is still too small to provide precise estimates of a modest effect of early intensive BP lowering on IVH. 
In summary, early intensive BP lowering according to the INTERACT protocol had no clear effect on IVH growth, although the lowest IVH growth was achieved in patients with sustained BP control over $24 \mathrm{~h}$.

\section{Acknowledgment}

The INTERACT studies were supported by research grants from the National Health and Medical Research Council (NHMRC) of Australia, which played no role in study design, conduct, analyses, or reporting.

\section{Disclosure Statement}

T. Robinson reports consultancy payments from Bayer, Boehringer Ingelheim and Daiichi Sankyo, and his institution has received grant funding from the National Institute of Health Research, the British Heart Foundation, the Stroke Association, and the Engineering and Physical Sciences Research Council. J. Chalmers reports grants from the NHMRC during the conduct of the study, grants from Servier International outside the submitted work, and being a chief or co-chief investigator for other large stroke trials. C.S. Anderson reports grant support from the NHMRC, advisory board fees from Astra Zeneca and Medtronic, and speaker fees from Takeda China. The remaining authors have nothing to disclose.

\section{References}

1 Chan E, Anderson CS, Wang X, Arima H, Saxena A, Moullaali TJ, Heeley E, Delcourt C, Wu G, Wang J, Chen G, Lavados PM, Stapf C, Robinson T, Chalmers J, Huang Y; INTERACT2 Investigators: Significance of intraventricular hemorrhage in acute intracerebral hemorrhage: Intensive Blood Pressure Reduction in Acute Cerebral Hemorrhage Trial results. Stroke 2015;46:653-658.

-2 Anderson CS, Heeley E, Huang Y, Wang J, Stapf C, Delcourt C, Lindley R, Robinson T, Lavados P, Neal B, Hata J, Arima H, Parsons M, Li Y, Wang J, Heritier S, Li Q, Woodward M, Simes RJ, Davis SM, Chalmers J; INTERACT2 Investigators: Rapid blood-pressure lowering in patients with acute intracerebral hemorrhage. N Engl J Med 2013;368:2355-2365.

-3 Ohwaki K, Yano E, Nagashima H, Hirata M, Nakagomi T, Tamura A: Blood pressure management in acute intracerebral hemorrhage: relationship between elevated blood pressure and hematoma enlargement. Stroke 2004;35:1364-1367.

4 Anderson CS, Huang Y, Wang JG, Arima H, Neal B, Peng B, Heeley E, Skulina C, Parsons MW, Kim JS, Tao QL, Li YC, Jiang JD, Tai LW, Zhang JL, Xu E, Cheng Y, Heritier S, Morgenstern LB, Chalmers J; INTERACT Investigators: Intensive Blood Pressure Reduction in Acute Cerebral Haemorrhage Trial (INTERACT): a randomised pilot trial. Lancet Neurol 2008; 7:391-399.

5 Fortes Lima TT, Prandini MN, Gallo P, Cavalheiro S: Prognostic value of intraventricular bleeding in spontaneous intraparenchymal cerebral hemorrhage of small volume: a prospective cohort study. Neurosurgery 2012;70:929-934.

6 Carcel C, Wang X, Sato S, Stapf C, Sandset EC, Delcourt C, Arima H, Robinson T, Lavados P, Chalmers J, Anderson CS; INTERACT2 Investigators: Degree and timing of intensive blood pressure lowering on hematoma growth in intracerebral hemorrhage: Intensive Blood Pressure Reduction in Acute Cerebral Hemorrhage Trial-2 results. Stroke 2016;47:1651-1653.

7 Moullaali TJ, Sato S, Wang X, Rabinstein AA, Arima H, Carcel C, Chen G, Robinson T, Heeley E, Chan E, Delcourt C, Stapf C, Cordonnier C, Lindley RI, Chalmers J, Anderson CS; INTERACT Investigators: Prognostic significance of delayed intraventricular haemorrhage in the INTERACT studies. J Neurol Neurosurg Psychiatry 2016, Epub ahead of print. 